\title{
Penile prosthesis in the management of erectile dysfunction following cancer therapy
}

\author{
Pranav Dadhich, Mark Hockenberry, E. Will Kirby, Larry Lipshultz \\ Department of Urology, Baylor College of Medicine, Houston, Texas, USA \\ Contributions: (I) Conception and design: M Hockenberry, L Lipshultz (II) Administrative support: P Dadhich; (III) Provision of study materials or \\ patients: P Dadhich, M Hockenberry; (IV) Collection and assembly of data: P Dadhich, M Hockenberry; (V) Data analysis and interpretation: All \\ authors; (VI) Manuscript writing: All authors; (VII) Final approval of manuscript: All authors. \\ Correspondence to: Larry Lipshultz, MD. Chief, Division of Male Reproductive Medicine and Surgery, Scott Department of Urology, Baylor College \\ of Medicine, 6624 Fannin Street, Suite 1700, Houston, TX 77030, USA. Email: larryl@bcm.edu.
}

\begin{abstract}
Erectile dysfunction (ED) is a common problem in older men and occurs with even greater frequency following the treatment of pelvic malignancies. Inflatable penile prosthesis (IPP) implantation is a safe and effective form of definitive ED treatment for those men who fail more conservative measures, and it can be used with similar outcomes in men following cancer therapy. Although many of these men remain dissatisfied with other therapeutic options for ED, IPPs are underutilized in this population. This review will discuss the current practice patterns, outcomes and nuances to surgical technique regarding the use of IPPs in patients with ED following cancer therapy.
\end{abstract}

Keywords: Penile prosthesis; erectile dysfunction (ED); cancer therapy

Submitted Mar 30, 2017. Accepted for publication Jun 21, 2017.

doi: $10.21037 /$ tau.2017.07.05

View this article at: http://dx.doi.org/10.21037/tau.2017.07.05

\section{Introduction}

Erectile dysfunction (ED) continues to be a common problem following the treatment of prostate cancer and other pelvic malignancies. Despite improvements in surgical technique and radiation targeting, a significant percentage of patients suffer worsening erectile function after treatment. Fortunately, many therapeutic modalities exist. The initial management for ED involves oral medications due to availability, ease of use, and acceptable efficacy. A substantial number of patients, however, may be dissatisfied with the effectiveness of oral medications and frequently will progress through intraurethral suppositories, vacuum erection devices and intracavernosal injections. In many instances, patients fail to satisfactorily respond to these treatment options, given the potentially severe vascular, neurologic, histologic and anatomic alterations resulting from cancer therapy. Surgical implantation of an inflatable penile prosthesis (IPP) offers an effective and reliable solution to definitively treat ED in these cases.
Longitudinal data supports the efficacy of IPP implantation, and continued research in the field continues to advance the quality of the devices themselves as well as surgical techniques for implantation. Current information indicates, though, that this therapy is underutilized. A study by Tal et al. demonstrated that only $0.78 \%$ of men receive an IPP following radical prostatectomy or external beam radiation therapy for prostate cancer (1). This review will discuss the current practice patterns, outcomes and nuances to surgical technique regarding the use of IPPs in patients with ED following cancer therapy.

\section{ED incidence following cancer therapy}

ED commonly occurs following local treatment for prostate and other pelvic cancers. The direct and indirect mechanisms by which pelvic surgery and radiation impair erectile function has been extensively reviewed (2). Furthermore, these malignancies most commonly affect older men, who may suffer from decreased erection quality 
due to aging and are consequently more vulnerable to further vascular or neurological insult. Currently the median age of diagnosis is 66 and 73 years old for prostate and bladder cancer, respectively (3). Much work has been devoted to improving sexual outcomes following treatments for these malignancies, most notably the development of nerve-sparing radical prostatectomy (4) and conformal intensity modulated radiation therapy (5). Despite these advances, ED remains common after these therapies.

The epidemiology of ED following prostate cancer therapy has been most extensively investigated relative to the treatment of other pelvic malignancies due to the relatively high incidence of prostate cancer. In 2016, prostate cancer ranked third among newly diagnosed cancers, and it is estimated that $12.9 \%$ of men are diagnosed with prostate cancer in their lifetimes with 129.4 new cases diagnosed per 100,000 men annually (3). Wide ranges of erectile function following prostate cancer therapy exist in the literature due to patient self-reported outcomes and varying ED definitions.

The Prostate Intervention Versus Observation Trial (PIVOT) provides insight into erectile function following surgery. PIVOT enrolled 731 men with localized prostate cancer who were randomized to undergo either radical prostatectomy or observation. The study found that two years after surgery, $81 \%$ of patients reported ED, which was defined as the inability to achieve an erection sufficient for vaginal penetration. Conversely, the rate of ED in the control group assigned to active surveillance rather than surgery was only $44 \%$, significantly less than the surgical cohort (6). Furthermore, a later study by Nelson et al. demonstrated that only $4 \%$ of men above age 60 returned to baseline erectile function two years after RP (7).

Unlike the immediate effects of surgical therapy, the effects of radiation are delayed, and patients may experience a gradual rather than immediate decline in erectile function. Pinkawa $e t a l$. found that in patients undergoing external beam radiation therapy their ability to achieve an erection firm enough for intercourse progressively worsened over time. At 16 months post-therapy, only $27 \%$ reported achieving erections sufficiently firm for intercourse (8). Although less extensively studied, treatment of other pelvic malignancies, such as bladder cancer, rectal cancer and various sarcomas, have similar negative effects on erectile function. Surgical removal of the bladder also involves removal of the prostate utilizing dissection similar to that of radical prostatectomy. Therefore, similar nerve disruption can occur with radical cystectomy relative to radical prostatectomy.
Relatively rare literature exists on nerve-sparing during radical cystectomy. Although concrete estimates of ED after radical cystectomy are not well described, diminished sexual function is prevalent after this surgery (9).

Current data indicates that the prevalence of sexual dysfunction remains high as well in patients undergoing surgery for rectal cancer. Hendren et al. found that, for men following surgery at a median follow-up of 58 months, 69\% of International Index of Erectile Function (IIEF) scores indicated ED (defined as one standard deviation below the average IIEF obtained) and $93 \%$ reported some new sexual problem after surgery (10). Although little is currently known regarding the effects on erectile function of pelvic radiation for bladder or rectal cancer, the negative effect on erections is likely similar to prostate radiation. Unfortunately, it is clear that men treated for pelvic malignancies have very high rates of ED overall and significantly greater likelihood of ED than other men.

\section{Progression to IPP following cancer therapy}

Many men suffer from significant ED following cancer therapy, and as a result a large proportion of those men experience distress and decreased quality of life (11). Management of ED typically follows a stepwise progression from less invasive to more invasive modalities until sufficient erections for the patient's satisfaction are achieved. Initial treatment entails medical therapy with oral phosphodiesterase type 5 inhibitors (PDE5i). The efficacy of these medications have been well established in general with a majority of patients exhibiting variable positive responses to therapy (12). Within a population of post-prostate cancer treatment patients specifically, however, outcomes are inferior. A recent review shows that about one half of men using PDE5i therapy after prostate cancer treatment were unable to achieve erections sufficient for sexual intercourse; patients who experienced severe ED exhibited the weakest responses to the medications (13). Consequently, many men need to progress to more invasive therapy to effectively treat their ED.

The Prospective Registry of Outcomes with Penile Prosthesis for Erectile Restoration (PROPPER) study shows that RP remains the most common primary etiology for ED in men undergoing IPP placement. $28 \%$ of men in the registry underwent placement of IPP following radical prostatectomy (14). Despite accounting for the majority of patients undergoing IPP placement, the utilization of IPP in this post-cancer therapy population remains extremely low overall. According to Surveillance, Epidemiology and End 
Results data, only $0.78 \%$ of 68,500 men receiving treatment for prostate cancer eventually underwent IPP placement (1). A small difference was noted following different treatment modalities with $2.3 \%$ of men following radical prostatectomy patients receiving an IPP compared to $0.3 \%$ of men following radiation. Predictors of IPP insertion included younger age and lack of marriage (1). These findings are consistent with a study by Stephenson and colleagues that followed patients for up to 5 years after either RP or radiation therapy and demonstrated an IPP utilization rate of $1.9 \%$ following cancer treatment (15). Together these data and the high percentage of patients who fail medical management following cancer therapy indicate a vast underutilization of IPP to treat ED in these patients. The excellent outcomes obtained with IPP in this population further underscore the need to improve utilization.

\section{IPP outcomes}

IPP continues to be a highly efficacious and reliable option to help any patient with ED achieve excellent quality erections. Patients with IPP have reported higher degrees of satisfactory erectile function when compared to patients receiving medical management. Analysis from Rajpurkar et al. demonstrated that men who received an IPP had significantly higher scores on all measured indices of erectile function when compared to medically managed patients. Notably, the erectile function domain of the IIEF was over $37 \%$ higher in patients with an IPP when compared to those medically managed (16). Furthermore, the long-term mechanical efficacy of IPP has been well established. Data show that about $60 \%$ of IPPs will be free of mechanical failures for 15 years after implantation (17). Device technology continues to advance and will likely further improve outcomes (17). Although fewer studies have examined IPP outcomes in patients following specific cancer therapy, the efficacy, satisfaction and reliability appear similar. Lane et al. demonstrated safe and successful IPP placement in 115 patients after radical prostatectomy without significant intraoperative complications. Furthermore, at a median follow-up of 3 years, the estimated probabilities of infection and mechanical failure remained low at $3 \%$ and $4.5 \%$, respectively (18). Similar IPP outcomes are obtained following pelvic radiation as well. Dubocq et al. followed patients who received an IPP after prostate radiation and found that no infections or erosions had occurred at a mean follow-up of 40 months (19).

Cancer therapy, both surgery and radiation, can create challenges to prosthetic placement; these nuances must be recognized by implanters to optimize outcomes. More specifically, cancer therapy can change anatomy in such a way to complicate placement of the IPP reservoir due to disruption of the space of Retzius or of the IPP cylinders due to corporal fibrosis and curvature. Additionally, stress incontinence is a common comorbidity of prostatectomy. Treatment may require implantation of an artificial urinary sphincter (AUS) either before or in combination with an IPP. Lastly, cancer therapy may increase the rare risk of postoperative IPP complications, such as reservoir erosion into adjacent structures. A preoperative awareness of these potentially complicating factors enables more successful IPP implantation.

\section{Nuances to IPP implantation following cancer therapy}

\section{Reservoir placement}

In the setting of virgin anatomy, the IPP reservoir is traditionally placed in the space of Retzius, the extraperitoneal space posterior to the pubic bone and anterior to the bladder. This maneuver is usually done through a bluntly developed defect in the transversalis fascia, which forms the floor of the inguinal canal at the external inguinal ring. Although rare, complications may occur despite normal anatomy due to close proximity to major structures. The external iliac vein is $2.5-4 \mathrm{~cm}$ away from the external inguinal ring, as demonstrated in a study of 25 cadavers, and may be inadvertently damaged while the decompressed bladder (5.3-8 $\mathrm{cm}$ away from the ring) may also be mistakenly entered (20). This analysis reinforces avoidance of dissection lateral to the external inguinal ring and ensuring bladder decompression prior to reservoir placement. Cancer therapy can create an environment of scarring and diminished tissue quality that can heighten the risks of these injuries by requiring more force to achieve the same dissection and increasing tissue susceptibility to damage (21).

Physical disruption of the space of Retzius, and its subsequent operative challenges to IPP placement, remains a concern unique to surgical therapy. IPP placement following robotic-assisted laparoscopic prostatectomy (RALP), in particular, presents a significant challenge and higher complication risk, as the retropubic space is eliminated during this intraperitoneal surgical approach to mobilize the bladder and access the prostate. Fortunately, these complications are rare. The open radical 
prostatectomy dissection is confined to the extraperitoneal space and consequently leaves the peritoneum intact to serve as a barrier to intraperitoneal structures during future IPP reservoir placement. Following RALP, therefore, intraperitoneal structures, such as bowel, may also be in close proximity to the external inguinal ring and more easily injured during reservoir placement. This issue has become especially relevant as RALP accounts for over $70 \%$ of prostatectomies relative to the once traditional open approach (22). A poll of sexual medicine practitioners found that IPP reservoir placement in the retropubic space is either sometimes (46\%) or frequently (36\%) harder to place following RALP (22).

Device modifications and alternative techniques have been developed to circumvent this problem. An initial solution entailed removing the reservoir entirely from the IPP and the creation of a two-piece inflatable implant comprised only of cylinders and a control pump. These two-piece implants remain a viable alternative in patients with particularly hostile pelvises with low rates of mechanical failure and an $85 \%$ patient satisfaction rate (23). Two-piece implants are unable to achieve the same rigidity, though, as the standard three-piece IPPs, which includes a reservoir. More recently, alternate sites for reservoir placement outside of the space of Retzius (ectopic placement) have been used to avoid risk of damage to intraperitoneal structures.

The majority of ectopic reservoir placements are submuscular, referring to a position posterior to the rectus abdominis muscle, while other possibilities include subcutaneous and lateral retroperitoneal placement. In 2002, Wilson et al. first described successful ectopic reservoir placement in the space anterior to the transversalis fascia and posterior to rectus abdominis entered with finger or instrument through the superior wall of the inguinal canal at the external inguinal ring (24). This positioning anterior to the transversalis fascia has benefits beyond avoiding an obliterated retropubic space and can also prevent other postoperative complications such as reservoir erosion into the bladder and compression of the external iliac vein (21). Drawbacks to the ectopic location, however, include an increased likelihood of palpation in the abdominal wall and of cylinder autoinflation due to increased pressure around the reservoir. Device improvements by both IPP manufacturers, Boston Scientific and Coloplast, have minimized these downsides with the advent of low profile reservoirs and fluid lock-out valves (25). Reservoir palpability was noted in only $3.4 \%$ of 2,687 patients, and autoinflation occurred in only $0.09 \%$ of patients in the same series of ectopic submuscular placement reviewed by Stembler et al. (26). Complications in that series were also rare with $0.09 \%$ of patients experiencing bladder injury and $1.4 \%$ experiencing reservoir hernia (26). Subsequent technical improvements include a high submuscular approach pioneered by Morey et al. to avoid palpability and reservoir herniation. In this technique, a submuscular space is created more cephalad from the external inguinal ring using a Foerster lung-grasping clamp (27). Patient self-reported satisfaction with IPP following the high submuscular approach for reservoir placement was $96 \%$ in a series of 96 patients (28). Based on such data, the United States Food and Drug Administration approved the ectopic placement of the Coloplast IPP reservoir in 2015 (25).

\section{Penile modeling and curvature correction}

Following either surgical or radiation therapy, significant scarring and fibrosis of involved tissues can occur. At times, fibrotic penile plaques can develop and contribute to a significant curvature of the erection known as Peyronie's disease. Since this penile deformity becomes more noticeable with a rigid erection, this penile angulation may go unnoticed prior to therapy for ED due to inadequate erectile function following cancer therapy. Data has shown that the incidence of Peyronie's disease can be as high as $15.9 \%$ in men who previously underwent prostatectomy and later presented for sexual dysfunction (29). Although close to $90 \%$ of men with some penile curvature have the condition corrected with IPP inflation alone, awareness of significant curvature is important preoperatively when planning IPP insertion, as various additional techniques may be employed intraoperatively to aid correction (30). Segal et al. has described the use of manual modeling, tunical relaxation, tunical excision and, finally, plaque incision and grafting as a graded means for reducing curvature. In the patients studied, $22 \%$ required straightening maneuvers; of those, $47 \%$ underwent manual modeling, 37\% underwent tunical relaxation and $16 \%$ required tunical reconstruction. Although a $3 \%$ risk of urethral injury exists, the study showed no increase in adverse IPP outcomes in patients requiring these maneuvers (31).

\section{AUS and combined placement with IPP}

In addition to ED, patients who have undergone treatment for prostate cancer commonly also suffer from some degree of urinary incontinence. Most notably, compromise of the 
urethral sphincter after radical prostatectomy can create significant stress incontinence. Data from the PIVOT trial shows that at 2 years follow-up $17 \%$ of patients who underwent RP suffered from stress urinary incontinence or required an indwelling catheter (6). Patients suffering from post-prostatectomy incontinence have a number of options, including pelvic floor physical therapy, urethral sling or AUS placement. Kim and colleagues found that only about $6 \%$ of men underwent any sort of surgical procedures for post-prostatectomy incontinence (32). Similarly, Berge et al. demonstrated that only about $5 \%$ of patients underwent AUS placement after radical prostatectomy (33). Like IPP, placement of AUS appears significantly underutilized in this patient population. It seems intuitive that the patients counseled regarding urological prosthetics and willing to undergo one procedure to improve quality of life would also be interested in use of the additional prosthetic device for a concomitant problem. Consequently, IPP placement is often performed following or in combination with AUS placement.

The rates of mechanical failures, infections and erosions as well as average hospital stay do not significantly differ for a combined procedure when compared with placement of AUS or IPP alone. Furthermore, patients who did have complications with one device did not report simultaneous adverse outcomes in the other. Combined surgery can actually provide patients with numerous advantages, such as the possibility to perform the procedures with a single incision, a lower total operative time and lower total costs (31). During the combined surgery, AUS urethral cuff placement creates the greatest risk of aborting the procedure secondary to urethral injury. Thus, the protocol traditionally involves first placing the AUS cuff through a transperineal incision, followed by placement of the IPP through penoscrotal incision, and finally, placement of the AUS reservoir in the preperitoneal space (31). Placement of both IPP and AUS through a single transscrotal incision has been shown to be feasible; however, robust data do not exist regarding the outcomes of this simultaneous approach (34).

\section{Risk of erosion}

Regardless of proper IPP placement, the possibility of erosion still remains for both the cylinders and the reservoir. Previous pelvic surgery and radiation therapy are primary risk factors for erosion due to reduction in tissue quality combined with chronic pressure from device components that could result in ischemic necrosis. Reservoirs are at risk for erosion into the bladder or urinary diversions following cystectomy; however, the majority of erosive complications occur at the distal tips of prosthesis cylinders. Fortunately, the rate of reservoir erosion following cancer surgery is extremely low at $0.4 \%(35)$.

\section{Conclusions}

Diminished erectile function continues to be a common comorbidity in the treatment of genitourinary malignancies. Although some patients are adequately managed through medical therapy, many others can be better served through definitive surgical treatment. Current IPP and AUS utilization remains low despite the safety and efficacy of these procedures in this population. Urologists should be aware, however, of various technical nuances when performing IPP placement in men following cancer therapy.

\section{Acknowledgements}

None.

\section{Footnote}

Conflicts of Interest: The authors have no conflicts of interest to declare.

\section{References}

1. Tal R, Jacks LM, Elkin E, et al. Penile implant utilization following treatment for prostate cancer: analysis of the SEER-Medicare database. J Sex Med 2011;8:1797-804.

2. Annam K, Voznesensky M, Kreder KJ. Understanding and Managing Erectile Dysfunction in Patients Treated for Cancer. J Oncol Pract 2016;12:297-304.

3. Surveillance, Epidemiology, and End Results (SEER) Program Research Data (1973-2013), National Cancer Institute, DCCPS, Surveillance Research Program, Surveillance Systems Branch, released April 2016, based on the November 2015 submission. Available online: www.seer.cancer.gov

4. Walsh PC, Lepor H, Eggleston JC. Radical prostatectomy with preservation of sexual function: Anatomical and pathological considerations. Prostate 1983;4:473-85.

5. Brown MW, Brooks JP, Albert PS, et al. An analysis of erectile function after intensity modulated radiation therapy for localized prostate carcinoma. Prostate Cancer Prostatic Dis 2007;10:189-93.

6. Wilt TJ, Brawer MK, Jones KM, et al. Radical 
prostatectomy versus observation for localized prostate cancer. N Engl J Med 2012;367:203-13.

7. Nelson CJ, Scardino PT, Eastham JA, et al. Back to baseline: erectile function recovery after radical prostatectomy from the patients' perspective. J Sex Med 2013;10:1636-43.

8. Pinkawa M, Gagel B, Piroth MD, et al. Erectile dysfunction after external beam radiotherapy for prostate cancer. Eur Urol 2009;55:227-34.

9. Modh RA, Mulhall JP, Gilbert SM. Sexual dysfunction after cystectomy and urinary diversion. Nat Rev Urol 2014;11:445-53.

10. Hendren SK, O'Connor BI, Liu M, et al. Prevalence of Male and Female Sexual Dysfunction Is High Following Surgery for Rectal Cancer. Ann Surg 2005;242:212-23.

11. Chambers SK, Chung E, Wittert G, et al. Erectile dysfunction, masculinity, and psychosocial outcomes: a review of the experiences of men after prostate cancer treatment. Transl Androl Urol 2017;6:60-8.

12. Scaglione F, Donde S, Hassan TA, et al. Phosphodiesterase Type 5 Inhibitors for the Treatment of Erectile Dysfunction: Pharmacology and Clinical Impact of the Sildenafil Citrate Orodispersible Tablet Formulation. Clin Ther 2017;39:370-7.

13. Candy B, Jones L, Williams R, et al. Phosphodiesterase type inhibitors in the management of erectile dysfunction secondary to treatments for prostate cancer: Findings from a Cochrane systematic review. BJU Int 2008;102:426-31.

14. Henry GD, Karpman E, Brant W, et al. The Who, How and What of Real-World Penile Implantation in 2015: The PROPPER Registry Baseline Data. J Urol 2016;195:427-33.

15. Stephenson RA, Mori M, Hsieh YC, et al. Treatment Of Erectile Dysfunction Following Therapy For Clinically Localized Prostate Cancer: Patient Reported Use And Outcomes From The Surveillance, Epidemiology, And End Results Prostate Cancer Outcomes Study. J Urol 2005;174:646-50.

16. Rajpurkar A, Dhabuwala CB. Comparison of satisfaction rates and erectile function in patients treated with sildenafil, intracavernous prostaglandin E1 and penile implant surgery for erectile dysfunction in urology practice. J Urol 2003;170:159-63.

17. Wilson SK, Delk JR, Salem EA, et al. Long-term survival of inflatable penile prostheses: single surgical group experience with 2,384 first-time implants spanning two decades. J Sex Med 2007;4:1074-9.

18. Lane BR, Abouassaly R, Angermeier KW, et al. Three-piece inflatable penile prostheses can be safely implanted after radical prostatectomy through a transverse scrotal incision. Urology 2007;70:539-42.

19. Dubocq FM, Bianco FJ Jr, Maralani SJ, et al. Outcome analysis of penile implant surgery after external beam radiation for prostate cancer. J Urol 1997;158:1787-90.

20. Henry G, Hsiao W, Karpman E, et al. A guide for inflatable penile prosthesis reservoir placement: pertinent anatomical measurements of the retropubic space. J Sex Med 2014;11:273-8.

21. Simon R, Hakky T, Henry GD, et al. Tips and tricks of inflatable penile prosthesis reservoir placement: a case presentation and discussion. J Sex Med 2014;11:1325-33.

22. Karpman E, Sadeghi-Nejad H, Henry G, et al. Current opinions on alternative reservoir placement for inflatable penile prosthesis among members of the sexual medicine society of North America. J Sex Med 2013;10:2115-20.

23. Lux M, Reyes-Vallejo L, Morgentaler A, et al. Outcomes and satisfaction rates for redesigned penile prosthesis. $\mathrm{J}$ Urol 2007;177:262-6.

24. Wilson SK, Henry GD, Delk JR, et al. The Mentor Alpha I penile prosthesis with reservoir lock-out valve: effective prevention of auto-inflation with improved capability for ectopic reservoir placement. J Urol 2002;168:1475-8.

25. Hakky T, Lentz A, Sadeghi-Nejad H, et al. The evolution of the inflatable penile prosthesis reservoir and surgical placement. J Sex Med 2015;12:464-7.

26. Stember DS, Garber BB, Perito PE. Outcomes of abdominal wall reservoir placement in inflatable penile prosthesis implantation: a safe and efficacious alternative to the space of retzius. J Sex Med 2014;11:605-12.

27. Morey AF, Cefalu CA, Hudak SJ. High submuscular placement of urologic prosthetic balloons and reservoirs via transscrotal approach. J Sex Med 2013;10:603-10.

28. Chung PH, Morey AF, Tausch TJ, et al. High Submuscular Placement of Urologic Prosthetic Balloons and Reservoirs: 2-Year Experience and Patient-reported Outcomes. Urology. 2014;84:1535-40.

29. Tal R, Heck M, Teloken P, et al. Peyronie's disease following radical prostatectomy: incidence and predictors. J Sex Med 2010;7:1254-61.

30. Mulhall J, Ahmed A, Anderson M. Penile Prosthetic Surgery for Peyronie's Disease: Defining the Need for Intraoperative Adjuvant Maneuvers. J Sex Med 2004;1:318-21.

31. Segal RL, Cabrini MR, Harris ED, et al. Combined inflatable penile prosthesis-artificial urinary sphincter implantation: no increased risk of adverse events compared to single or staged device implantation. J Urol 
2013;190:2183-8.

32. Kim PH, Pinheiro LC, Atoria CL, et al. Trends in the use of incontinence procedures after radical prostatectomy: a population-based analysis. J Urol 2013;189:602.

33. Berge V, Thompson T, Blackman D. Additional surgical intervention after radical prostatectomy, radiation therapy, androgen-deprivation therapy or watchful waiting. Eur

Cite this article as: Dadhich P, Hockenberry M, Kirby EW, Lipshultz L. Penile prosthesis in the management of erectile dysfunction following cancer therapy. Transl Androl Urol 2017;6(Suppl 5):S883-S889. doi: 10.21037/tau.2017.07.05
Urol 2007;52:1036.

34. Wilson S, Delk J 2nd, Henry GD, et al. New surgical technique for sphincter urinary control system using upper transverse scrotal incision. J Urol 2003;169:261-4.

35. Tran CN, Boncher N, Montague DK, et al. Erosion of Inflatable Penile Prosthesis Reservoir into Neobladder. J Sex Med 2013;10:2343-6. 